\title{
A NOTE ON START-UP, PLANE COUETTE FLOW INVOLVING SECOND-GRADE FLUIDS
}

\author{
P. M. JORDAN
}

Received 30 August 2004 and in revised form 3 December 2004

We point out and correct several errors that appear in a recently published paper on transient flows of second-grade fluids.

\section{Introduction}

In a recent work, Hayat et al. [4] considered several transient flows of a second-grade (SG) fluid (see $[3,6,8]$ and the references therein). Included in their study was the case of a SG fluid undergoing start-up Couette flow between two infinite parallel plates. (Here, we should mention that various versions of this problem for SG fluids have been studied $[1,5,9]$.) Unfortunately, a detailed examination of their solution (i.e., [4, equation (16)]) reveals that it is incorrect. Moreover, this error is propagated throughout [4, Section 4] since $[4$, equation (16)] is used to compute other quantities which characterize the flow.

The main aims of the present note are the following: (i) to point out some of these inaccuracies; (ii) give the correct expressions; (iii) provide additional analytical insight into this problem; and (iv) present numerical work in support of (i)-(iii).

\section{Problem formulation}

To this end, we begin by noting that, with a few additions, the same notation, coordinate system, and so forth used in [4] are also used in the present work. Now, taking the positive $y$-axis of a Cartesian coordinate system in the upward direction, let an incompressible, homogeneous, SG fluid fill the slab $y \in(0, h)$ between two flat, infinite solid plates that occupy the planes $y=0, h$. Initially, both the fluid and the plates are at rest. At $t=0^{+}$, the fluid is set in motion by the sudden acceleration of the top plate, in a direction parallel to the $x$-axis, to a constant velocity $U(\neq 0)$; that is, the velocity of the top plate is given by $(U H(t), 0,0)$, where $H(\cdot)$ denotes the Heaviside unit step function. Neglecting all body forces and observing that the pressure gradient is zero since the pressure can be at most a function of time only [5], we wish to determine the flow at every point in the slab for all $t>0$.

By translational invariance in the $x z$-plane, the velocity vector has the form $\mathbf{v}=(u(y$, $t), 0,0)$. As a result, the continuity equation $\nabla \cdot \mathbf{v}=0$ is identically satisfied and $\dot{\mathbf{v}}=$ $(\partial u(y, t) / \partial t, 0,0)$, where a superposed dot denotes the material time derivative. Thus, 
the mathematical model of the flow consists of the following initial-boundary value problem (IBVP):

$$
\begin{gathered}
\frac{\partial u}{\partial t}=v \frac{\partial^{2} u}{\partial y^{2}}+\alpha \frac{\partial^{3} u}{\partial t \partial x^{2}}, \quad(y, t) \in(0, h) \times(0, \infty) \\
u(0, t)=0, \quad u(h, t)=U H(t), \quad t>0 ; \quad u(y, 0)=0, \quad y \in(0, h) .
\end{gathered}
$$

Here, the positive constant $\nu$ is the kinematic viscosity and $\alpha=\alpha_{1} / \rho$, where the positive constants $\alpha_{1}$ and $\rho$ denote the first normal stress moduli and the density, respectively [3].

\section{Exact solution using the Laplace transform}

Employing the nondimensional independent variables $\eta \equiv y / h$ and $\tau \equiv v t / h^{2}$ (see [4, Figure 1]) and then applying the temporal Laplace transform [2] to (2.1) and the boundary conditions (BCs), we obtain, after employing the initial condition (IC) and solving the resulting subsidiary equation, the transform domain solution

$$
U^{-1} \bar{u}(\eta, s)=\frac{1}{s}\left\{\frac{\sinh \left[\eta \sqrt{s /\left(1+s l^{2}\right)}\right]}{\sinh \left[\sqrt{s /\left(1+s l^{2}\right)}\right]}\right\}
$$

where $s$ denotes the transform parameter, a bar over a quantity denotes its image in the transform domain, and we let $l \equiv h^{-1} \sqrt{\alpha}$. Observing that the singular points of $\bar{u}(\eta, s)$ are simple poles located at

$$
s_{n}=\frac{-n^{2} \pi^{2}}{1+l^{2} n^{2} \pi^{2}} \quad(n=0,1,2,3, \ldots)
$$

we invert (3.1) by using the residue theorem to evaluate the Laplace inversion integral [2]. This yields, after simplifying, the exact $\eta \tau$-domain series solution

$$
U^{-1} u(\eta, \tau)=H(\tau)\left\{\eta+\frac{2}{\pi} \sum_{n=1}^{\infty} \exp \left[\frac{-n^{2} \pi^{2} \tau}{1+l^{2} n^{2} \pi^{2}}\right] \frac{(-1)^{n} \sin [n \pi \eta]}{n\left(1+l^{2} n^{2} \pi^{2}\right)}\right\} .
$$

Equation (3.3), which is given by Jordan and Puri [5], is the correct solution to IBVP (2.1)-(2.2). It differs from [4, equation (16)] in that the denominator of each term in the series appearing in the former is $n\left(1+l^{2} n^{2} \pi^{2}\right)$, whereas in the case of the latter, the corresponding denominators contain only $n$. In the same way, [4, equation (17)], which is taken directly from [1, equation (9.5)], is also incorrect. In this case, the correct expression is

$$
U^{-1} u(\eta, \tau)=H(\tau)\left\{(1-\eta)-\frac{2}{\pi} \sum_{n=1}^{\infty} \exp \left[\frac{-n^{2} \pi^{2} \tau}{1+l^{2} n^{2} \pi^{2}}\right] \frac{\sin [n \pi \eta]}{n\left(1+l^{2} n^{2} \pi^{2}\right)}\right\} .
$$

\section{Analytical results}

4.1. Temporal limits. Having just obtained the correct solution to IBVP (2.1)-(2.2), we now examine the small-and large-time behavior of $u$. Below are listed the limiting values 
of $u$ as $\tau$ approaches zero (from above) and infinity, respectively. (Clearly, the $\tau \rightarrow 0^{-}$ limit of $u$ is identically zero.)

$$
U^{-1} \lim _{\tau \rightarrow 0^{+}} u(\eta, \tau)=\left\{\frac{\sinh [\eta / l]}{\sinh [1 / l]}\right\}, \quad U^{-1} \lim _{\tau \rightarrow \infty} u(\eta, \tau)=\eta,
$$

where $\eta \in(0,1)$ is assumed fixed. These limits were derived from (3.1) using the relations $\lim _{\tau \rightarrow 0^{+}} u(\eta, \tau)=\lim _{s \rightarrow \infty} s \bar{u}(\eta, s)$ and $\lim _{\tau \rightarrow \infty} u(\eta, \tau)=\lim _{s \rightarrow 0} s \bar{u}(\eta, s)[5,2]$.

4.2. Start-up jump discontinuity. As was noted in [5], the velocity field in this case exhibits a stationary jump discontinuity, which could also be called a nonpropagating vortex sheet [7], across the plane $\tau=0$, that is, at start-up. The magnitude of this jump, which is defined here as $[u] \equiv \lim _{\tau \rightarrow 0^{+}} u(\eta, \tau)-\lim _{\tau \rightarrow 0^{-}} u(\eta, \tau)$, is given by

$$
\left|\frac{[u]}{U}\right|=\left\{\frac{\sinh [\eta / l]}{\sinh [1 / l]}\right\}, \quad \eta \in(0,1) .
$$

In addition, we remark that dipolar fluids, a class of non-Newtonian fluids of which SG fluids can be considered a special case, also exhibits this type of jump at start-up [5].

4.3. Small-time expression. Recasting (3.1) in terms of exponentials and then expanding for large $s$ yields, after neglecting terms on $O\left[s^{-3}\right]$ and simplifying,

$$
\begin{aligned}
U^{-1} \bar{u}(\eta, s) \approx & \frac{1}{s} \sum_{n=0}^{\infty} \exp \left[\frac{-(2 n+1-\eta)}{l}\right] \exp \left[\frac{\nu(2 n+1-\eta)}{2 l \alpha s}\right]\left\{1-\frac{3 \nu^{2}(2 n+1-\eta)}{8 h^{4} l^{5} s^{2}}\right\} \\
& -\frac{1}{s} \sum_{n=0}^{\infty} \exp \left[\frac{-(2 n+1+\eta)}{l}\right] \exp \left[\frac{\nu(2 n+1+\eta)}{2 l \alpha s}\right]\left\{1-\frac{3 v^{2}(2 n+1+\eta)}{8 h^{4} l^{5} s^{2}}\right\} .
\end{aligned}
$$

Inverting term by term using a table of inverses [2] and Bessel function identities, we obtain the correct small-time series approximation

$$
\begin{aligned}
U^{-1} u(\eta, \tau) \approx \sum_{n=0}^{\infty} \exp \left[\frac{-(2 n+1-\eta)}{l}\right]\left\{I_{0}\right. & {\left[\sqrt{\left.\frac{2(2 n+1-\eta) \tau}{l^{3}}\right]}\right.} \\
& \left.-\frac{3 \tau}{4 l^{2}} I_{2}\left[\sqrt{\frac{2(2 n+1-\eta) \tau}{l^{3}}}\right]\right\} \\
-\sum_{n=0}^{\infty} \exp \left[\frac{-(2 n+1+\eta)}{l}\right]\left\{I_{0}\left[\sqrt{\left.\frac{2(2 n+1+\eta) \tau}{l^{3}}\right]}\right]\right. & \left.-\frac{3 \tau}{4 l^{2}} I_{2}\left[\sqrt{\frac{2(2 n+1+\eta) \tau}{l^{3}}}\right]\right\} \quad\left(\tau \ll l^{2}\right),
\end{aligned}
$$

where $\tau>0$ is assumed and $I_{\zeta}[\cdot]$ denotes the modified Bessel function of the first kind of order $\zeta$. 
4.4. Volume flux expression. Since [4, equation (16)] was used to determine [4, equation (19)], the latter is also incorrect. To obtain the correct expression for the volume flux, we integrate (3.3) with respect to $\eta$ from zero to one. This yields, after simplifying [5],

$$
\begin{aligned}
U^{-1} Q(\tau)=H(\tau)\{ & \frac{1}{2}-\frac{1}{\pi^{2}} \sum_{m=0}^{\infty} \exp \left[\frac{-4 \pi^{2}(m+1 / 2)^{2} \tau}{1+4 l^{2} \pi^{2}(m+1 / 2)^{2}}\right] \\
& \left.\times \frac{1}{(m+1 / 2)^{2}\left\{1+4 l^{2} \pi^{2}(m+1 / 2)^{2}\right\}}\right\} .
\end{aligned}
$$

Here, we should mention that contrary to what is stated in [4], $Q \neq 0$ when $\tau=0$, but is rather given by

$$
U^{-1} \lim _{\tau \rightarrow 0^{+}} Q(\tau)=l \tanh \left[(2 l)^{-1}\right],
$$

a result which can also be obtained directly from $(4.1)_{1}$.

\section{Numerical results}

In this section, we use the software package Mathematica (Version 5.0) to plot the velocity profiles $u / U$ versus $\eta$ for various fixed values of $\tau$. The plan here is the following. First, determine the number of terms needed in (3.3) to obtain a sufficiently converged result. Second, show that (3.3) and [4, equation (16)] are correct and incorrect, respectively, by comparing them against the numerically generated inverse of (3.1). And lastly, examine the behavior of the velocity field with respect to $\tau$ and $l$.

To this end, we have, in Figure 5.1, plotted (3.3) for various numbers of terms in the series. Clearly, this series converges very quickly and that taking only 100 terms yields extremely good results for the four values of $\tau$ considered. Hence, hereafter, we will always take 100 terms when evaluating (3.3).

Next, to verify our results, we have employed Tzou's [10] Riemann sum inversion algorithm (TRSIA) to numerically invert the Laplace transform domain solution given in (3.1). We executed TRSIA, which is given by

$$
u(\eta, \tau) \approx \frac{\exp [4.7]}{\tau}\left\{\frac{1}{2} \bar{u}\left(\eta, \frac{4.7}{\tau}\right)+\operatorname{Re}\left[\sum_{n=1}^{N}(-1)^{n} \bar{u}\left(\eta, \frac{4.7+\mathrm{i} n \pi}{\tau}\right)\right]\right\} \quad(\tau>0),
$$

where $N(\gg 1)$ is a positive integer and $\operatorname{Re}[\zeta]$ denotes the real part of the complex number $\zeta$, as a simple Mathematica program. As evident from Figure 5.2, the profiles plotted from (3.3) are in excellent agreement with those generated from TRSIA for all values of $\tau$ considered. In contrast, we see that the profiles corresponding to [4, equation (16)], which are plotted as the broken curves, are in agreement with the other two curves only for large values of $\tau$; that is, as $\tau$ is decreased, the error in [4, equation (16)] becomes more apparent. In fact, for $\tau=0.01$, the profile corresponding to [4, equation (16)] appears to be forming a jump discontinuity, and thus cannot represent a solution to IBVP (2.1)-(2.2). 


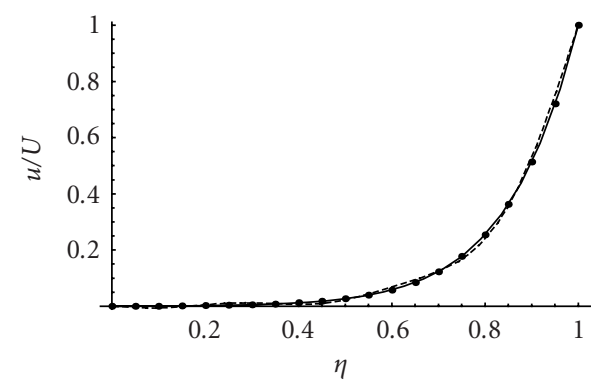

(a)

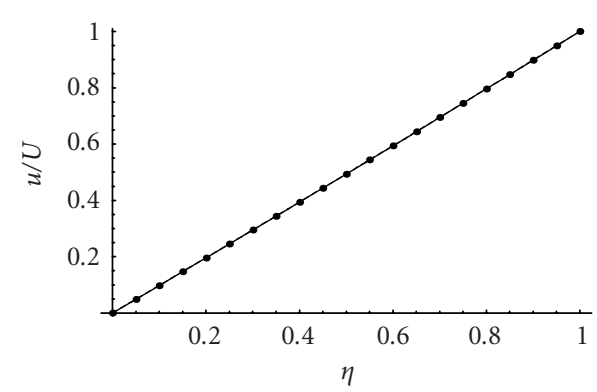

(c)

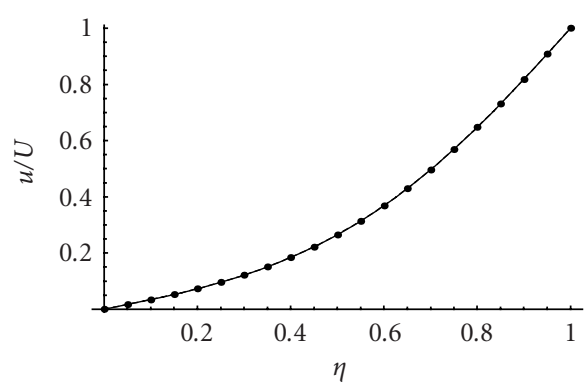

(b)

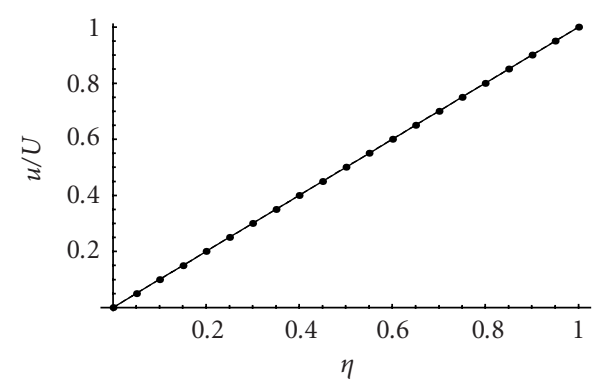

(d)

Figure 5.1. $u / U$ versus $\eta$ from (3.3) for $\tau=$ (a) 0.01 , (b) 0.1 , (c) 0.5 , (d) 1 , and $l=\sqrt{0.01}$. Dots: 10,000 terms, solid: 100 terms, and broken: 5 terms.

In Figure 5.3, we have illustrated the temporal evolution of the velocity profile for fixed l. As can also be inferred analytically, we see that $u$ is an increasing function of $\tau$ that is bounded above and below by

$$
\eta>\frac{u}{U}>\frac{\sinh [\eta / l]}{\sinh [1 / l]}
$$

Finally, in Figure 5.4, we have examined the behavior of $(4.1)_{1}$ as $l$ is varied. Here, we clearly see that the $\tau \rightarrow 0^{+}$limiting case of $u$ is an increasing function of $l$.

\section{Summary}

In this work, we have reexamined the problem of unsteady Couette flow involving SG fluids. We have pointed out a recent case in the literature in which the Laplace transformbased solution to this problem has been stated incorrectly and we have provided the needed correction(s). In addition, we have given the temporal limits of the velocity field, briefly discussed the nonpropagating vortex sheet associated with this particular SG flow, 


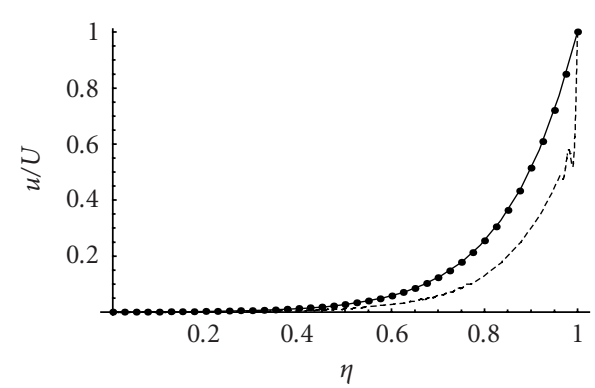

(a)

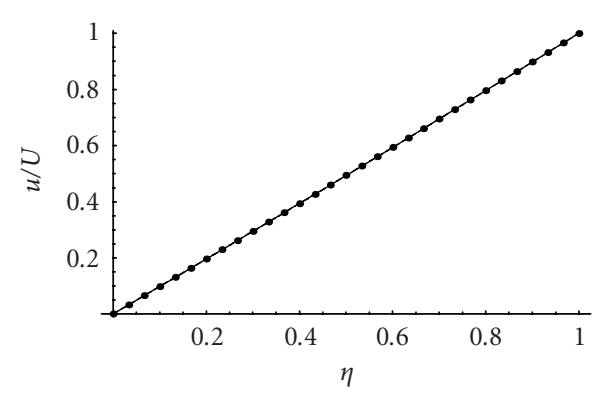

(c)

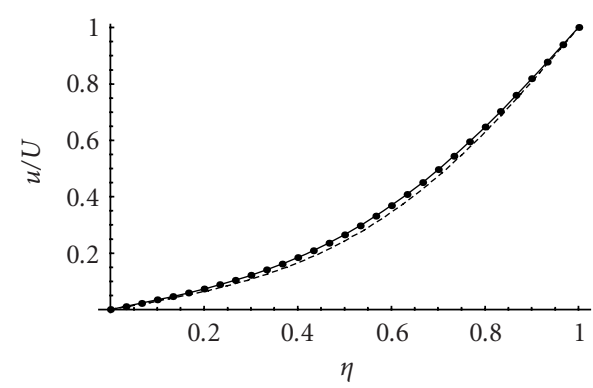

(b)

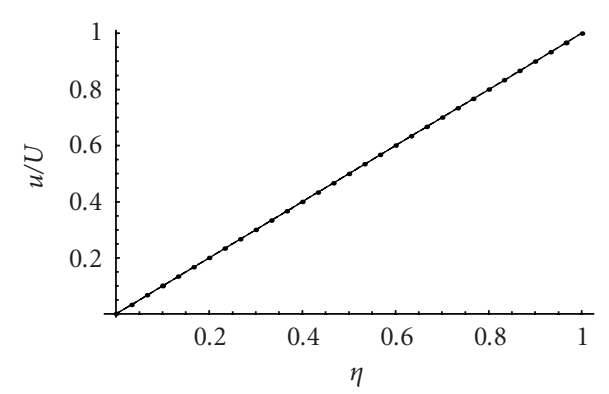

(d)

Figure 5.2. $u / U$ versus $\eta$ for $\tau=$ (a) 0.01 , (b) 0.1 , (c) 0.5 , (d) 1 , and $l=\sqrt{0.01}$. Solid: (3.3), dots: (5.1) with $N=10,000$, and broken: equation (16) in [4] (100 terms taken).

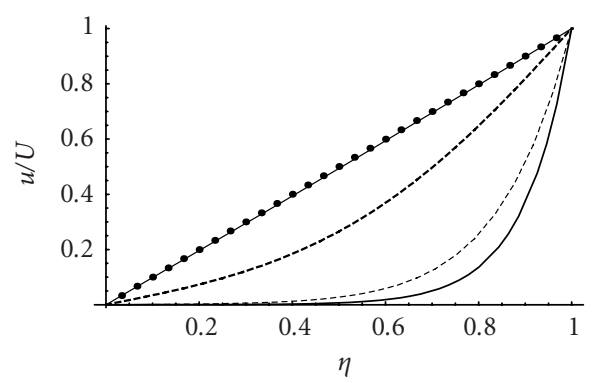

Figure 5.3. $u / U$ versus $\eta$ for $l=\sqrt{0.01}$. Bold: $(4.1)_{1}$, thin broken: (3.3) for $\tau=0.01$, bold broken: (3.3) for $\tau=0.1$, thin solid: (3.3) for $\tau=0.5$, and dots: (3.3) for $\tau=1$.

and have provided the correct small-time and volume flux expressions. What's more, velocity profile plots corresponding to a hypothetical SG fluid were also presented and we verified our solution numerically using TRSIA (see (5.1)). We have also listed several earlier works which addressed various cases of Couette flow involving SG fluids. 


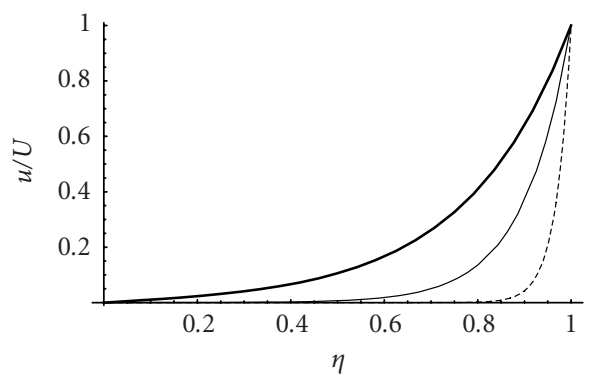

Figure 5.4. $u / U$ versus $\eta$ from $(4.1)_{1}$. Bold: $l=\sqrt{0.05}$, thin solid: $l=\sqrt{0.01}$, and broken: $l=\sqrt{0.001}$.

It should be noted that [4, equation (21)], which gives the friction force on the upper (i.e., moving) plate, is also incorrect. The correct form of this expression can be obtained by substituting (3.3) into [4, equation (20)]. For other analytical and numerical results regarding this problem, see [5]. And lastly, it must be stressed that, except for those discussed in the present note, the author has not checked, nor does he know for certain, if any of the other expressions given in [4] are incorrect.

\section{Acknowledgments}

The author was supported by ONR/NRL funding (PE 061153N). The author is indebted to Professor K. R. Rajagopal for his valuable suggestions.

\section{References}

[1] R. Bandelli and K. R. Rajagopal, Start-up flows of second grade fluids in domains with one finite dimension, Internat. J. Non-Linear Mech. 30 (1995), no. 6, 817-839.

[2] H. S. Carslaw and J. C. Jaeger, Operational Methods in Applied Mathematics, 2nd ed., Dover, New York, 1963.

[3] J. E. Dunn and K. R. Rajagopal, Fluids of differential type: critical review and thermodynamic analysis, Internat. J. Engrg. Sci. 33 (1995), no. 5, 689-729.

[4] T. Hayat, M. Khan, A. M. Siddiqui, and S. Asghar, Transient flows of a second grade fluid, Internat. J. Non-Linear Mech. 39 (2004), no. 10, 1621-1633.

[5] P. M. Jordan and P. Puri, Exact solutions for the unsteady plane Couette flow of a dipolar fluid, Proc. Roy. Soc. London Ser. A 458 (2002), no. 2021, 1245-1272.

[6] Stokes First Problem for a Rivlin-Ericksen fluid of second grade in a porous half-space, Internat. J. Non-Linear Mech. 38 (2003), no. 7, 1019-1025.

[7] R. E. Meyer, Introduction to Mathematical Fluid Dynamics, Dover, New York, 1982.

[8] K. R. Rajagopal, On boundary conditions for fluids of the differential type, Navier-Stokes Equations and Related Nonlinear Problems (Funchal, 1994) (A. Sequeira, ed.), Plenum, New York, 1995, pp. 273-278.

[9] T. W. Ting, Certain non-steady flows of second-order fluids, Arch. Rational Mech. Anal. 14 (1963), 1-26.

[10] D. Y. Tzou, Macro- to Microscale Heat Transfer: the Lagging Behavior, Taylor \& Francis, Washington, DC, 1997.

P. M. Jordan: Code 7181, Naval Research Laboratory, Stennis Space Center, MS 39529, USA

E-mail address: pjordan@nrlssc.navy.mil 


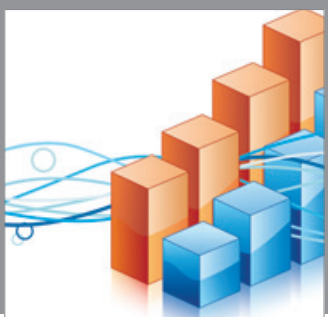

Advances in

Operations Research

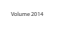

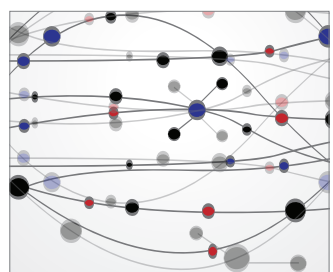

\section{The Scientific} World Journal
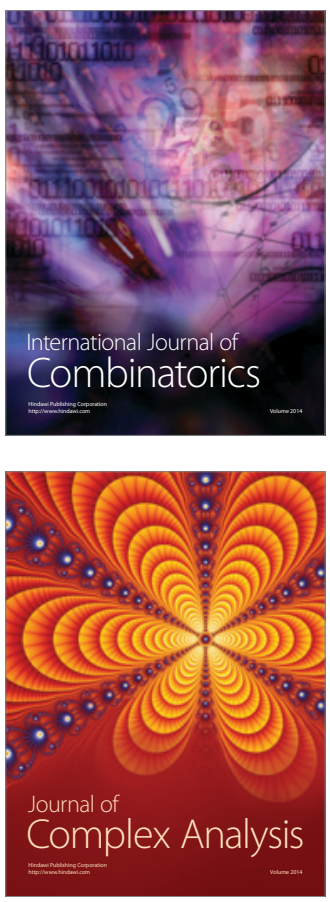

International Journal of

Mathematics and

Mathematical

Sciences
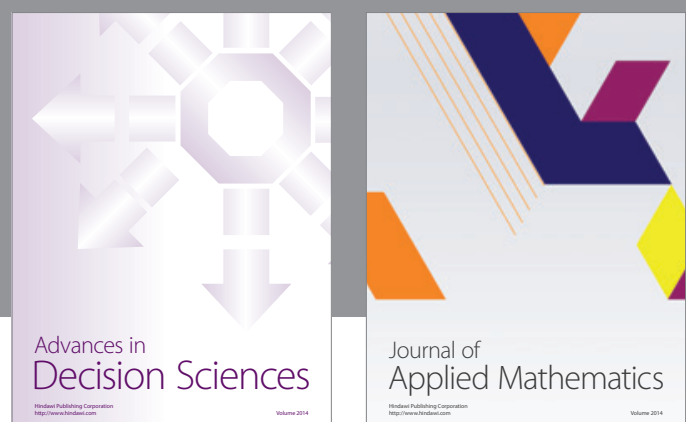

Journal of

Applied Mathematics
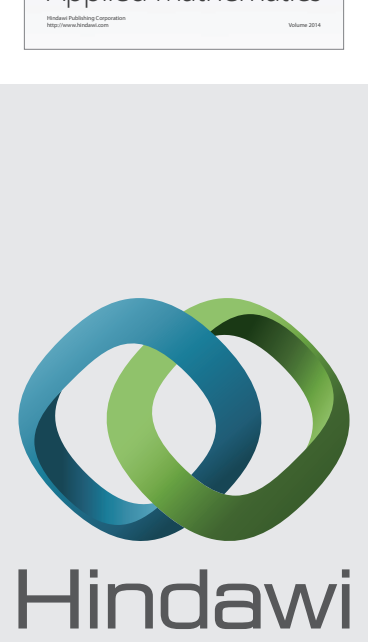

Submit your manuscripts at http://www.hindawi.com
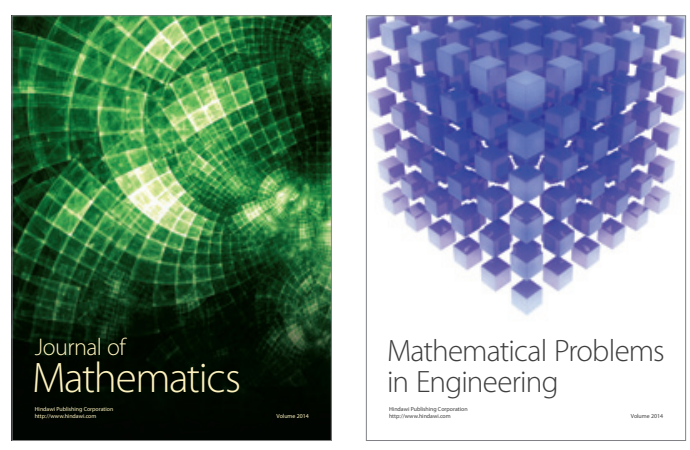

Mathematical Problems in Engineering
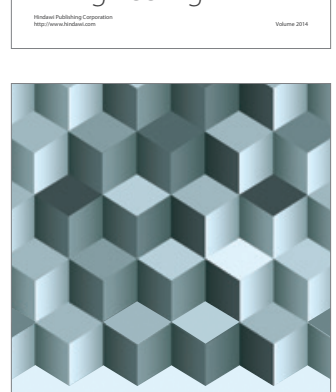

Journal of

Function Spaces
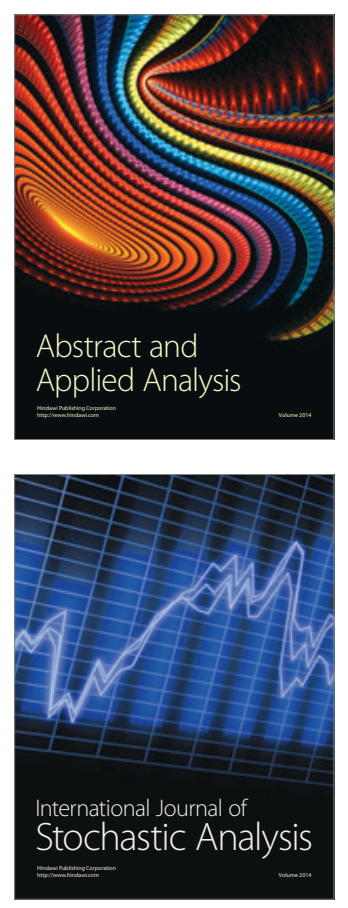

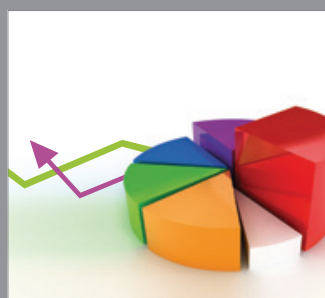

ournal of

Probability and Statistics

Promensencen
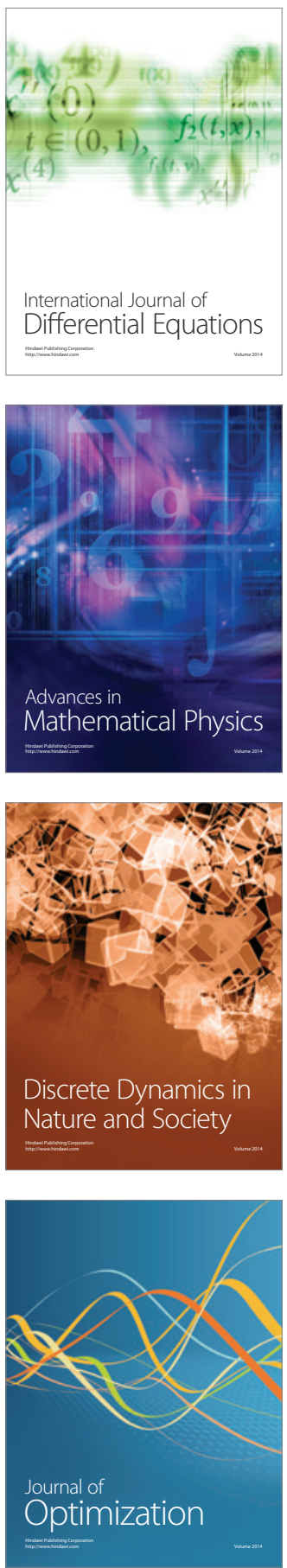